Please do not remove this page

RMIT

UNIVERSITY

\title{
A laser obstacle detection and avoidance system for manned and unmanned aircraft applications
}

Ramasamy, Subramanian; Gardi, Alessandro Giacomo Maria; Liu, Jing; Sabatini, Roberto

https://researchrepository.rmit.edu.au/esploro/outputs/9921862736701341/filesAndLinks?institution=61RMIT_INST\&index=null

Ramasamy, S., Gardi, A. G. M., Liu, J., \& Sabatini, R. (2015). A laser obstacle detection and avoidance system for manned and unmanned aircraft applications. Proceedings of the IEEE International Conference on Unmanned Aircraft Systems (ICUAS 2015), 526-533.

https://doi.org/10.1109/ICUAS.2015.7152332

Document Version: Accepted Manuscript

Published Version: https://doi.org/10.1109/ICUAS.2015.7152332

Repository homepage: https://researchrepository.rmit.edu.au

(C) 2015 IEEE

Downloaded On 2023/04/26 17:53:15 +1000

Please do not remove this page 
Thank you for downloading this document from the RMIT Research Repository.

The RMIT Research Repository is an open access database showcasing the research outputs of RMIT University researchers.

RMIT Research Repository: http://researchbank.rmit.edu.au/

\section{Citation:}

Ramasamy, S, Gardi, A, Liu, J and Sabatini, R 2015, 'A laser obstacle detection and avoidance system for manned and unmanned aircraft applications', in Proceedings of the IEEE International Conference on Unmanned Aircraft Systems (ICUAS 2015), United States, 9-12 June 2015, pp. 526-533.

See this record in the RMIT Research Repository at:

https://researchbank.rmit.edu.au/view/rmit:33432

Version: Accepted Manuscript

Copyright Statement: (C) 2015 IEEE

Link to Published Version:

http://dx.doi.org/10.1109/ICUAS.2015.7152332 


\title{
A Laser Obstacle Detection and Avoidance System for Manned and Unmanned Aircraft Applications
}

\author{
Subramanian Ramasamy, Alessandro Gardi, Jing Liu and Roberto Sabatini \\ School of Aerospace, Mechanical and Manufacturing Engineering \\ RMIT University, Melbourne, VIC 3000, Australia \\ roberto.sabatini@rmit.edu.au
}

\begin{abstract}
This paper presents the key design features, numerical simulations and experimental ground/flight test activities performed to verify the functionalities of an obstacle detection and avoidance system suitable for various classes of manned and unmanned aircraft. The Laser Obstacle Avoidance and Monitoring (LOAM) system is proposed as one of the key non-cooperative sensors adopted for avoiding obstacles/intruders in the context of a future Sense-and-Avoid (SAA) capability. After a brief description of the system architecture and of the main data processing algorithms, avoidance trajectory generation and performance estimation models are described. A simulation of the avoidance trajectory generation algorithm is performed in a realistic scenario. Additionally, a brief overview of ground and flight test activities performed on various platforms and their main results is also presented. Some of the key aspects of the LOAM Human Machine Interface and Interaction $\left(\mathrm{HMI}^{2}\right)$ design are also outlined. The demonstrated detection and avoidance performances and the robust trajectory generation algorithm ensure a safe avoidance of all classes of obstacles (i.e. ground and aerial) in all weather conditions and flight phases.
\end{abstract}

Keywords-Laser; Obstacle Avoidance; Sense-and-Avoid; Avoidance Trajectory; Human Machine Interface.

\section{INTRODUCTION}

A number of manned and unmanned aircraft safety-and mission-critical tasks involve low-level flight activities beyond the relatively safe aerodrome perimeter. The adoption of small-to-medium size Unmanned Aircraft (UA) in low-level or nap-of-the-earth flight missions has resulted in growing concerns regarding the overall safety of lives and property. In this context, obstacle detection, warning and avoidance capabilities are of paramount importance to ensure safety of aircraft flight operations. The outstanding angular resolution and accuracy characteristics of Light Detection and Ranging (LIDAR), coupled to its good detection performance in a wide range of incidence angles and weather conditions provide an ideal solution for obstacle detection and avoidance. The Laser Obstacle Avoidance and Monitoring (LOAM) system was originally developed and tested on rotary-wing platforms [1-4], and the development of a scaled version for small-to-medium size UA is also currently being performed [5-7]. The LOAM detects potentially dangerous obstacles that are in or nearby the nominal aircraft flight trajectory, classifies and stores the detected obstacles by using a history function. The LOAM system also provides guidance for optimal avoidance manoeuvres, as well as timely caution/warnings to the ground crew (both aural and visual).

\section{OPERATIONAL REQUIREMENTS}

The operational requirements for a reliable and effective obstacle and warning system are:

- Capability to detect all types of hazardous obstacles, including topographic features, vegetation, buildings, poles/masts, towers, cables and transmission lines.

- High minimum detection range, adequate for the platform to perform the required airspeed performances.

- Wide Field of View (FOV), adequate to completely cover the manoeuvring envelope limits of the platform.

- High range and bearing resolutions along with accuracies and good probability of detection to ensure that no real obstacle threat remains undetected.

- Operability in all-weather and all-time conditions.

- Very low false alarm rate, to prevent spurious warnings that would increase the pilot or Ground Control Station (GCS) pilot workload and prompt unnecessary avoidance manoeuvres, potentially disruptive to the overall safety of the carried out mission.

\section{ARCHITECTURE}

The key components of the LOAM are the Sensor Head Unit (SHU), the Processing Unit (PU), the Control Panel (CP) and the Display Unit (DU). An overall view of the SHU used in the original LOAM version for rotorcrafts is illustrated in Fig. 1. The laser beam originating in the LOAM SHU is electro-mechanically orientated to periodically scan the area around the nominal flight trajectory inside a Field of View (FOV) of $40^{\circ}$ in azimuth and $30^{\circ}$ in elevation, with an adjustable Field of Regard (FOR) capability of $\pm 20^{\circ}$ on azimuth, centred on the longitudinal axis of the platform. The resulting elliptical laser scanning pattern is represented in Fig. 2. As depicted in Fig. 2, during every full FOV scan (4 $\mathrm{Hz}$ refresh frequency), the LIDAR beam performs a number of elliptical scan patterns across the FOV. This scanning pattern is well suited to detect the most dangerous obstacles including wires due to the several and regularly spaced vertical lines that it produces. The overall LOAM integrated architecture is illustrated in Fig. 3. The PU, in particular, interacts with the UA Mission Management System (MMS) [8-10], which is integrated with the multisensory navigation system $[11,12]$ and the Sense-and- 
Avoid (SAA) system [13]. UA command and telemetry datalinks serve as the communication media between the platform and the ground systems.
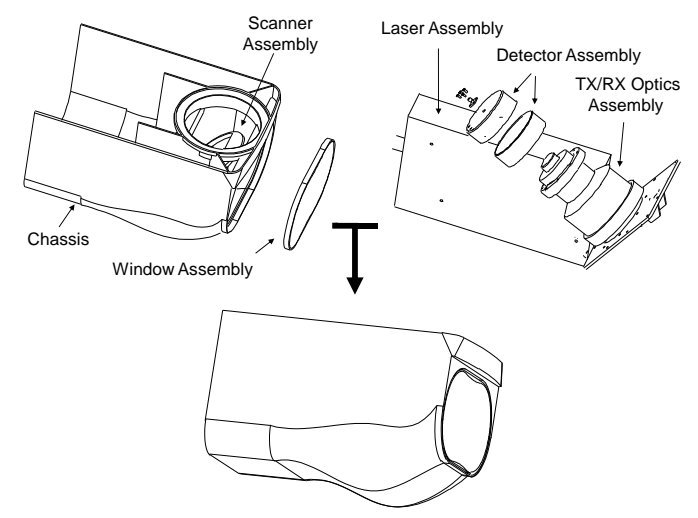

Fig.1. Illustration of the LOAM SHU. Adapted from [4].

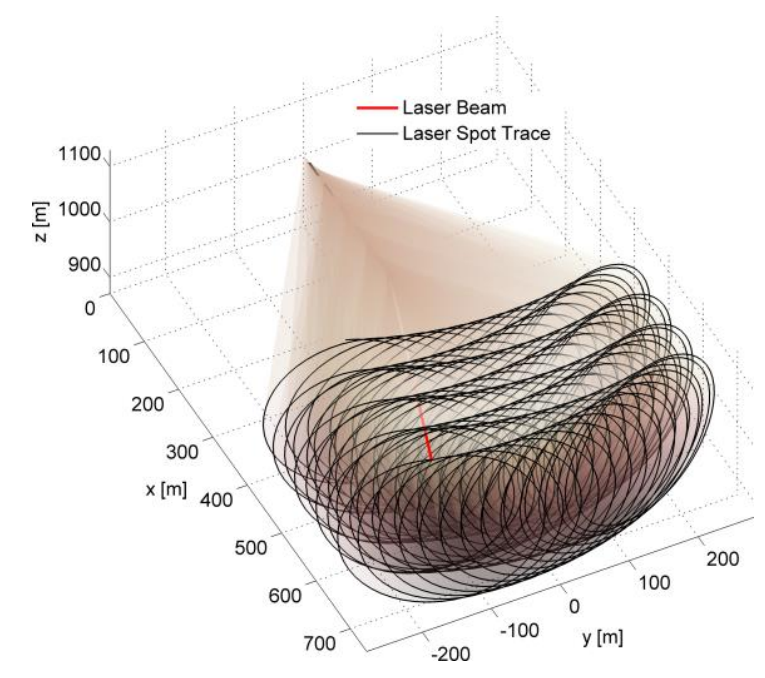

The signal processing software architecture is represented in Fig. 4. The LOAM performs echo detection through analogue signal processing that consists of an optical-electrical conversion, a signal pre-amplification and a threshold comparison. The low level processing algorithm processes only the echoes whose magnitude is weaker than pre-defined thresholds. The single echoes are processed as soon as they are acquired. The high level processing algorithm is based on the subset of acquired echoes in the current frame. Clusters are merged into a single obstacle by means of iterative image segmentation, specifically implemented to identify echoes characterised by uniform range. A statistical algorithm subsequently validates the merged echoes by verifying if the obstacle is generated by real aligned echoes or by noise data. The processing algorithms for extended obstacles are also divided in two different phases: echoes analysis and segmentation. The echoes already classified as extended objects need to be processed by a dedicated validation algorithm, since many of the detected signals are not generated by obstacles (like, for example, the ground).

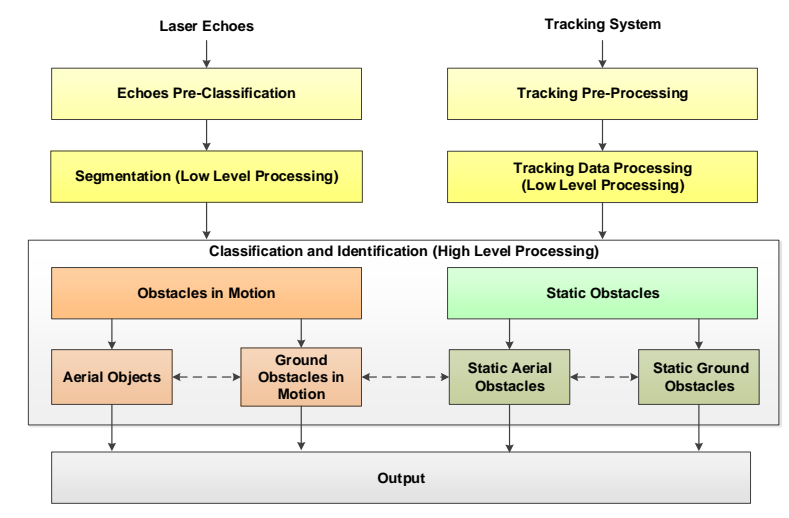

Fig. 4. LOAM signal processing software architecture.

Fig. 2. Scanning pattern of the LOAM laser beam.

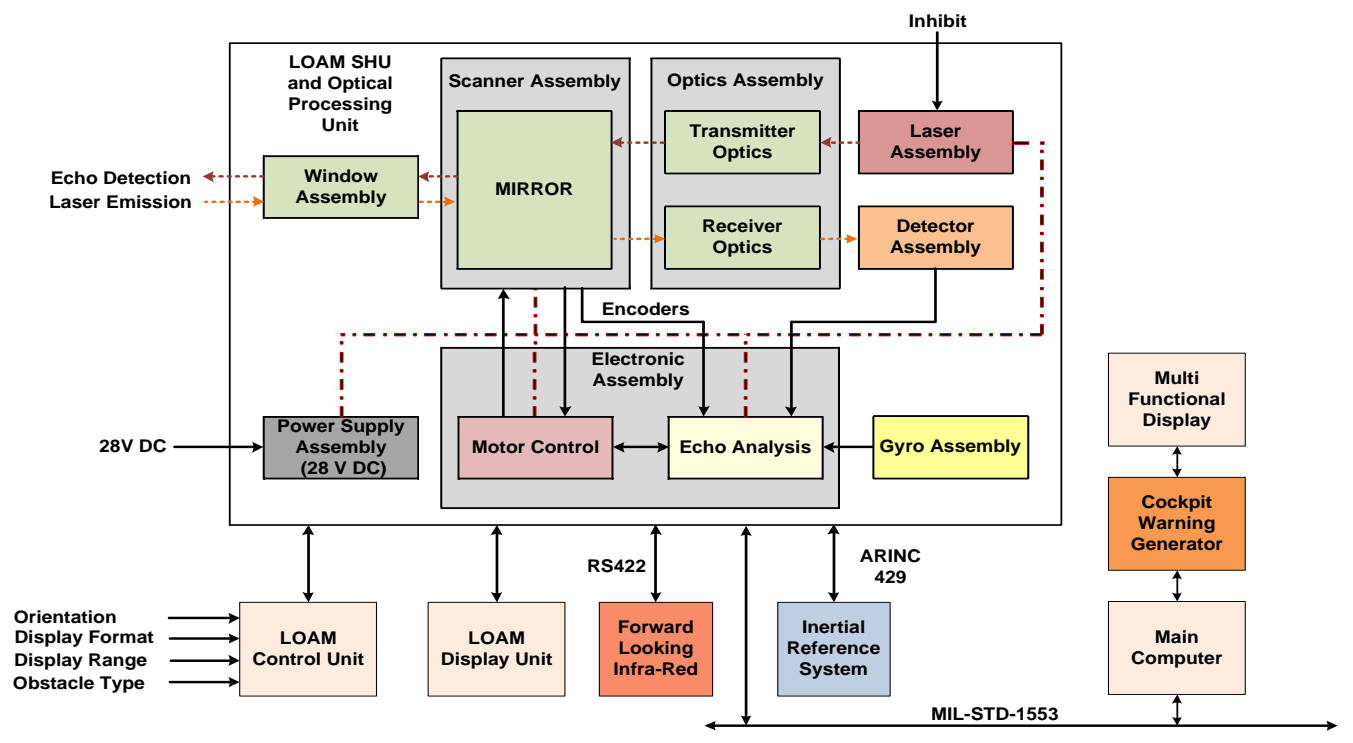

Fig. 3. LOAM hardware architecture showing the interfaces for avionics integration [7]. 
One of the main challenges in the integration of UA into non-segregated airspace is the SAA capability that enables the unmanned platforms to perform equally or exceed the performance of the see-and-avoid ability of the pilot in manned aircraft systems. Both cooperative and noncooperative SAA systems are being developed to enable UA to routinely access all classes of airspace In order to provide automated avoidance functionalities, the LOAM employs three key algorithms namely; prediction of the future platform trajectory, calculation of the potential collisions with the detected obstacles and generation of a set of optimal avoidance trajectories in case a risk of collision is determined. Identification and evaluation of the required sensors, as well as the associated data fusion algorithms, are a key constituent of the SAA system design. A number of cooperative systems and non-cooperative sensors can be employed in the SAA system design. The inclusion of ADS$B$ redefines the paradigm of Communication, Navigation and Surveillance (CNS) in Air Traffic Management (ATM) today by providing trajectory information. The noncooperative SAA sensors are employed to detect intruders or other obstacles in the UA Field of Regard (FOR) when cooperative systems are unavailable in the intruders. Optical, thermal, LOAM, Millimetre Wave (MMW) Radar and acoustic sensors are employed part of the noncooperative SAA system. The Standard Unified Method (SUM) of combining navigation and tracking uncertainties to determine the overall uncertainty volume surrounding an obstacle is introduced in [13].

\section{AVOIDANCE TRAJECTORY GENERATION}

The original avoidance trajectories generation algorithm for rotorcraft platforms was introduced in [4]. The algorithms for the generation of the avoidance trajectories in fixed-wing platforms were presented in [7]. Similarly to the implementation in rotorcrafts, the algorithm for fixed-wing aircraft is based on the dynamic programming approach. A direct optimisation method is implemented. Hence the implemented algorithm is based on the aircraft dynamics, and not on a geometric trajectory model. Robust decision logic is subsequently introduced to select the trajectory to be flied based on multiple criteria. The approximated dynamic model of the fixed-wing platform is based on a point-mass rigid body with three Degrees-of-Freedom (3-DoF) with constant mass. During the entire approach to the obstacle, the vehicle control system can provide a linear variation of $\mu$, up to the assumed maximum bank angle. This can be expressed as:

$$
\begin{cases}\mu=\mu_{0}+\dot{\mu}_{\mathrm{MAX}} \cdot \mathrm{t} & \left(\mu \leq \mu_{\mathrm{MAX}}\right) \\ \dot{\mu}=0 & \left(\mu=\mu_{\mathrm{MAX}}\right)\end{cases}
$$

The accuracy of 3-DoF flight dynamics has shown to be adequate for low-dynamics platforms, and in combination with smooth control logics leads to the generation of relatively smooth avoidance trajectories. Additional trajectory generation algorithms based on six Degrees-ofFreedom (6-DoF) dynamics are currently being developed, with aerodynamic and inertia coefficients. The maximum roll rate was set at $\mu_{\mathrm{MAX}}=20 \%$ s. The algorithms for estimation of the obstacle absolute motion based on differential geometry approach were introduced in [13]. In order to provide the fast and reliable performance required for our safety-critical task, the avoidance trajectory generation is based on simplified geometric shapes. The standard deviation of the LOAM detection and tracking error for each axis is given by:

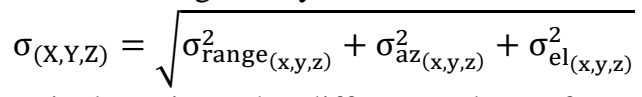

In particular, given the different values of uncertainty associated with the three cardinal directions, an ellipsoidal avoidance volume is implemented in the algorithm. In order to assure adequate safety levels, a separation buffer is introduced, which inflates the ellipsoidal avoidance volume associated with the obstacle. In particular, to provide a confidence level of $95 \%$, the uncertainty associated with the position of an obstacle is calculated as twice the standard deviation (i.e. the two-sigma) of the total obstacle detection and tracking errors. When the distance between two detected obstacles is comparable with the calculated uncertainty values, or with the UA dimensions, the algorithm combines the two obstacles in a single avoidance volume. The subsequent step involves the selection of the optimal trajectory from the generated set of safe trajectories, which is then fed to the aircraft guidance subsystems. The implemented decision logic is based on minimisation of the following cost function:

$$
J=w_{t} \cdot t_{S A F E}+w_{f} \int[S F C \cdot T(t)] d t-w_{d} \int D(t) d t
$$

where $t_{\mathrm{SAFE}}$ is the time at the point of minimum distance from the detected obstacles, hence it corresponds to the attainment of a safe condition, SFC $\left[\frac{\mathrm{kg}}{\mathrm{N}} \cdot \mathrm{s}\right]$ is the specific fuel consumption, $T(t)$ is the thrust profile, $D(t)$ is the distance from the ellipsoidal avoidance volume of the obstacle and $\mathrm{w}_{\mathrm{t}}, \mathrm{w}_{\mathrm{f}}, \mathrm{w}_{\mathrm{d}}$ are the weightings attributed to time, fuel and integral distance respectively. In time-critical avoidance applications (i.e., closing-up obstacles with high relative velocities and/or accelerations) appropriate higher weightings are used for the time and distance cost elements.

\section{Simulation AND Results}

Simulation activities were performed to validate the avoidance trajectory generation algorithm and to assess its performance. A realistic three-dimensional scenario for obstacle avoidance was introduced. The UA equipped with the LOAM is flying at an altitude $\mathrm{z}=100 \mathrm{~m}$ Above Ground Level (AGL) and approaching a number of obstacles. The original horizontal flight trajectory would lead to a collision with the obstacle. After a successful detection of all the potential dangerous obstacles, the algorithm calculates the distances among each of them. A representative set of avoidance trajectories generated following these assumptions, is depicted in Fig. 5. Fig. 6 illustrates the separation envelopes between the manned aircraft / UA and the boundary surface of the ellipsoidal avoidance volumes obtained from the uncertainty analysis described in [13], calculated for each point of the calculated trajectories. 


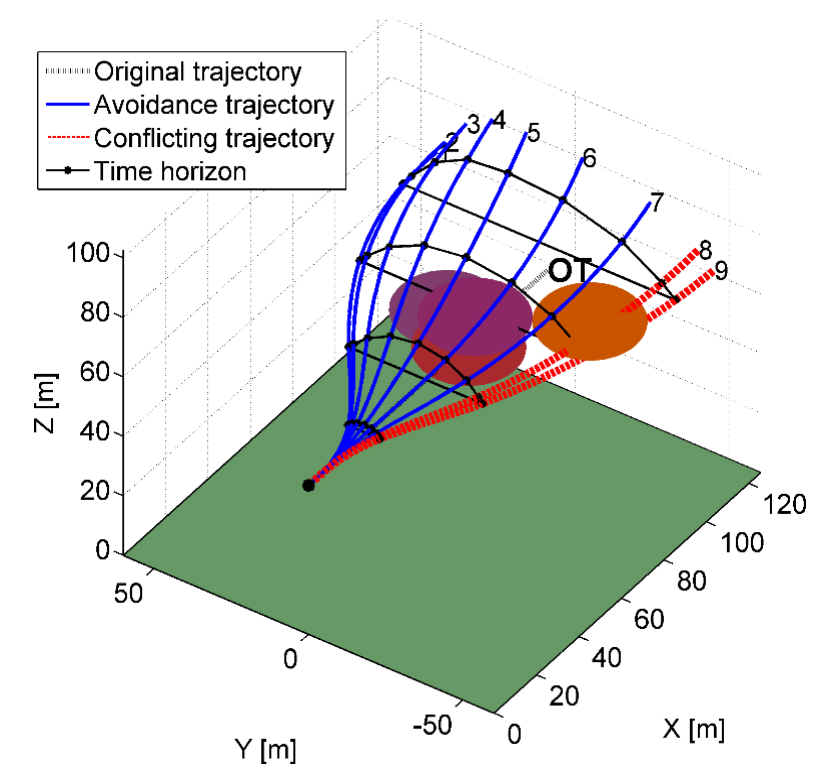

Fig. 5. LOAM avoidance trajectory generation.

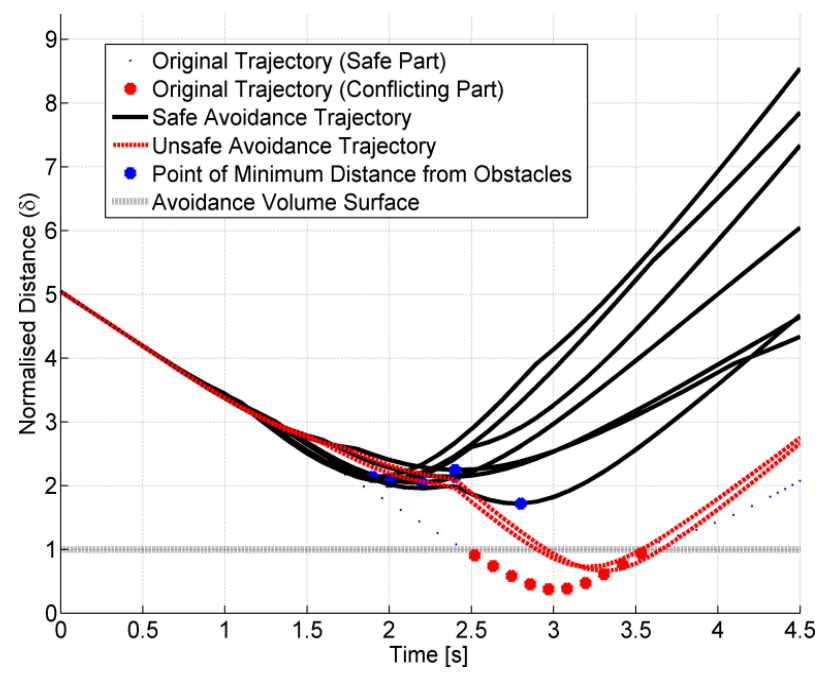

Fig. 6. Absolute distance of the trajectories from ellipsoidal avoidance volume boundaries surrounding the obstacles.

\section{HUMAN MACHINE INTERFACE AND INTERACTION}

Dedicated LOAM control and display interfaces are being developed for UA applications. Their characteristics are conceptually similar to the ones developed for the manned aircraft versions. However, as these interfaces are integrated in the Remote Piloting Station (RPS) and will be operated by the remote pilot, in this case the LOAM operating modes are activated using two different communication data links for Line-of-Sight (LOS) and Beyond LOS (BLOS) operations. For the purpose of Human Machine Interface (HMI) design, the human is modelled as an integral part of the Unmanned Aerial System (UAS) control loops and the adopted remote pilot model is illustrated in Fig. 7 [14-18]. The neuromuscular system is described by the below transfer function that is found to fit data obtained from experiments designed to isolate extrafusal muscle dynamics:

$$
\mathrm{H}_{\mathrm{md}}(\mathrm{s})=\frac{-\mathrm{K}_{\mathrm{md}} \mathrm{e}^{-\tau_{\mathrm{a}} \mathrm{s}}}{\left(1+\mathrm{T}_{\mathrm{N}} \mathrm{s}\right)\left(1+2 \xi_{\mathrm{a}} / \omega_{\mathrm{a}} \mathrm{s}+\mathrm{s}^{2} / \omega_{\mathrm{a}}^{2}\right)}
$$

where $K_{m d}$ is muscle dynamics describing function $\left(\mathrm{G}_{\mathrm{m}}\right)$ gain, $T_{N}$ is first-order lag time constant approximation of the neuromuscular system, $\xi_{a}$ is damping ratio of second-order term in $\mathrm{G}_{\mathrm{m}}, \omega_{a}$ is natural frequency of second-order term in $\mathrm{G}_{\mathrm{m}}$ and $\tau_{a}$ is time delay in $\mathrm{G}_{\mathrm{m}}$. The spindle feedback block is modelled using the following transfer function, which is effectively representing a delayed equalisation ability:

$$
\mathrm{H}_{\mathrm{sp}}(\mathrm{s})=\frac{\mathrm{K}_{\mathrm{sp}}\left(\mathrm{s}+\mathrm{Z}_{\mathrm{sp}}\right) \mathrm{e}^{-\tau_{\mathrm{sp}} \mathrm{s}}}{\mathrm{s}+\mathrm{P}_{\mathrm{sp}}}
$$

where $K_{s p}$ is spindle describing function gain, $Z_{s p}$ is spindle lead break frequency, $P_{s p}$ is spindle pole and $\tau_{s p}$ is spindle time delay. The joint sensor feedback block is the ensemble that represents the Golgi tendon organ feedback as well as various other modes of feedback that are difficult to isolate. It has been modelled by a simple gain and delay:

$$
\mathrm{H}_{\hat{\mathrm{j}}}(\mathrm{s})=\mathrm{K}_{\hat{\mathrm{j}}} \mathrm{e}^{-\tau_{\mathrm{j}} \mathrm{s}}
$$

where $K_{\hat{j}}$ is gain of effective joint feedback and $\tau_{\hat{j}}$ is time delay in joint angle feedback. The force feel system can be modelled using the following transfer function:

$$
\mathrm{Y}_{\mathrm{FS}}=1 /\left[\left(\mathrm{s} / \omega_{\mathrm{F}}\right)^{2}+2 \xi_{\mathrm{F}} \mathrm{s} / \omega_{\mathrm{F}}+1\right]
$$

where $\omega_{F}$ is feel system undamped natural frequency, $\xi_{F}$ is feel system damping ratio. A second order representation of the neuromuscular block was used in the studies and the following representation of the proprioceptive sensory model [19]:

$$
\mathrm{Y}_{\mathrm{PF}}=\mathrm{K}(\mathrm{s}+\mathrm{a}) \text { or } \mathrm{K} \text { or } \mathrm{K} /(\mathrm{s}+\mathrm{a})
$$

where $\mathrm{K}$ is a constant selected such that:

$$
\mathrm{Y}_{\mathrm{PF}}(\mathrm{s}) \propto \mathrm{s} \mathrm{Y}_{c}(\mathrm{~s})
$$

The aircraft dynamics is described by one of two transfer functions:

$$
\mathrm{Y}_{\mathrm{c}}=1 / \mathrm{s} \text { or } 1 / \mathrm{s}^{2}
$$

where Response Time $(\mathrm{RPT})=$ Reaction Time $(\mathrm{RT})+$ Movement Time (MT) and RT is given by:

$$
\mathrm{RT}=\mathrm{A}+\mathrm{B} \log _{2}(\mathrm{n}+1)
$$

where $\mathrm{n}$ is the number of choices presented to the human, and $\mathrm{A}$ and $\mathrm{B}$ are experimental constants and MT is given by:

$$
\mathrm{MT}=\mathrm{c}+\mathrm{dlog}_{2}(2 \mathrm{D} / \mathrm{W})
$$

where $\mathrm{c}$ and $\mathrm{d}$ are regression coefficients, $\mathrm{D}$ is movement distances and $\mathrm{W}$ is tolerance or width. 


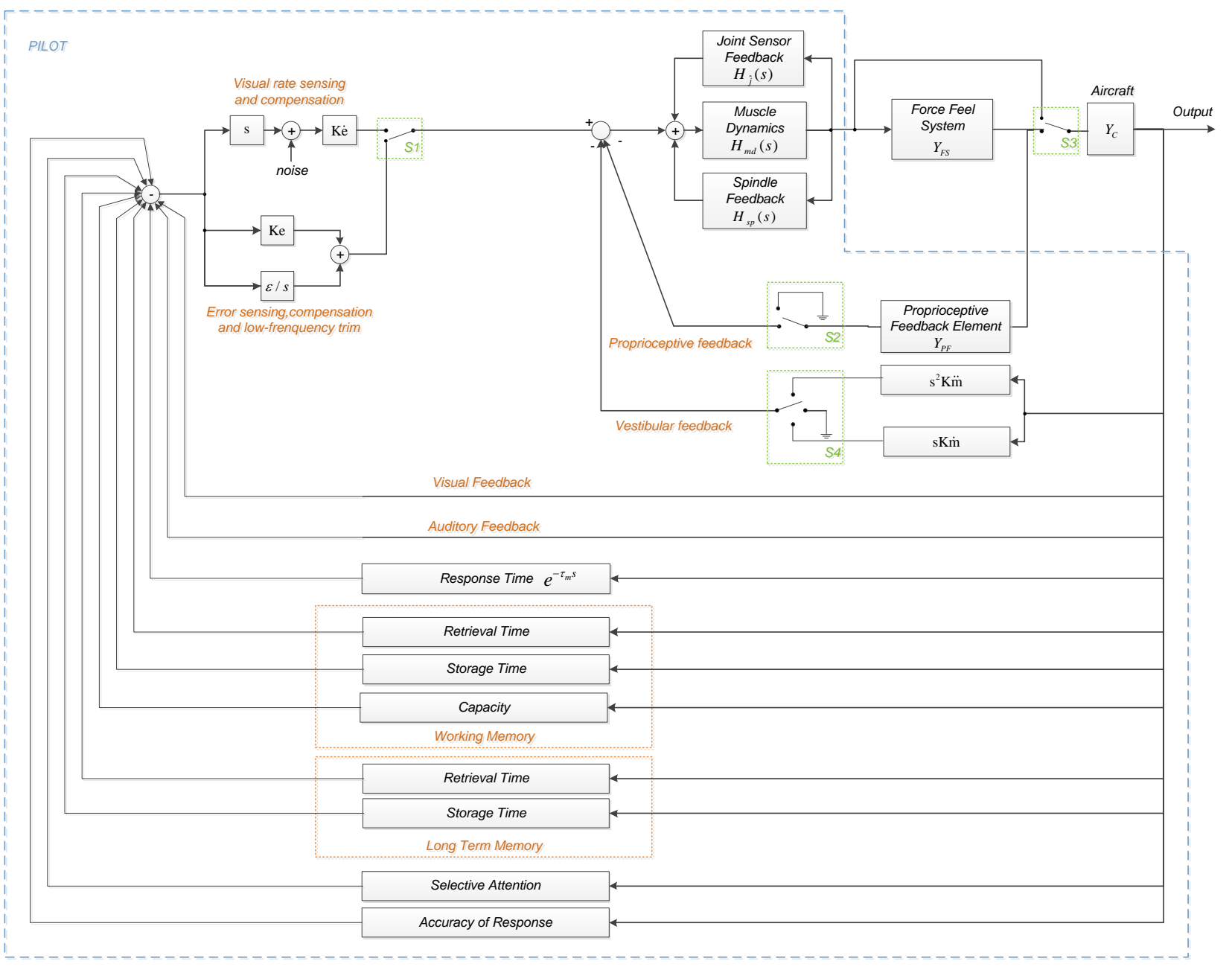

Fig. 7. Remote pilot model.

Different video outputs can be selected by the remote pilot according to the operational needs to properly display LOAM information and alerts. Different symbols are presented on the display units according to the characteristics of detected obstacles. The symbols used on UAS are a combination of the following:

- Safety Line.

- Wires.

- Pylon (vertical, linear obstacles e.g. poles and bare trees).

- Isolated obstacles (e.g. buildings, groups of trees, etc.).

- Distance from obstacle.

- OWS 3D (colour-coded LADAR image).

- Cautions.

- LOAM FOV.

- Obstacle warning.

- Flight vector.

- Evade advice cue.

- Plan Position Indicator (PPI).
The safety line (Fig. 8) is represented as the upper envelope of all detected obstacles and ground contours between minimum obstacle warning system detection range and the distance selected through the obstacle warning system control panel warning range selector.

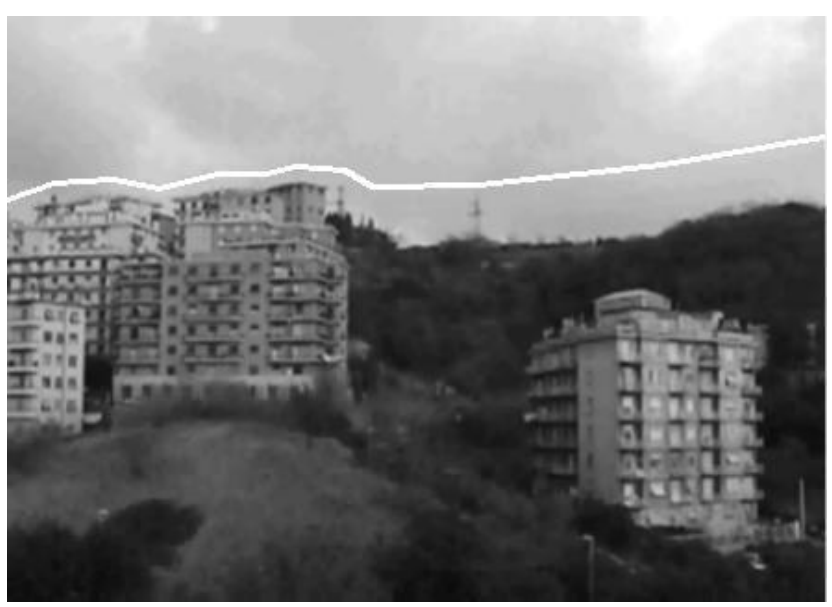

Fig. 8. An example of detection of safety line. 
The obstacle's distance is presented as a numeric value below the corresponding obstacle (Fig. 9). The numeric value is expressed in meter from helicopter present position.

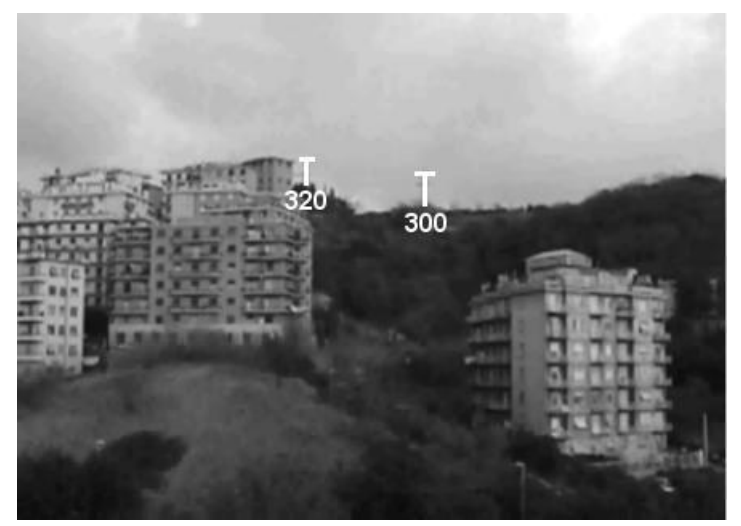

Fig. 9. An example of detection of safety line.

Fig. 10 shows some synthetic display formats currently being developed for the UAS applications. In particular, Fig. 10 depicts the safety line, which connects the points of minimum pitch for safe obstacle avoidance at all azimuths. Fig 10(b) depicts the synthetic vision format integrating information from the LOAM and from Forward Looking Sensor (FLS) systems.

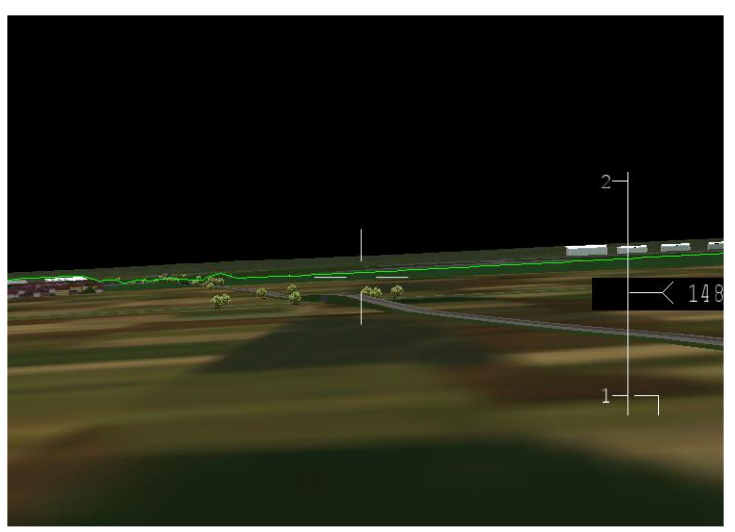

(a)

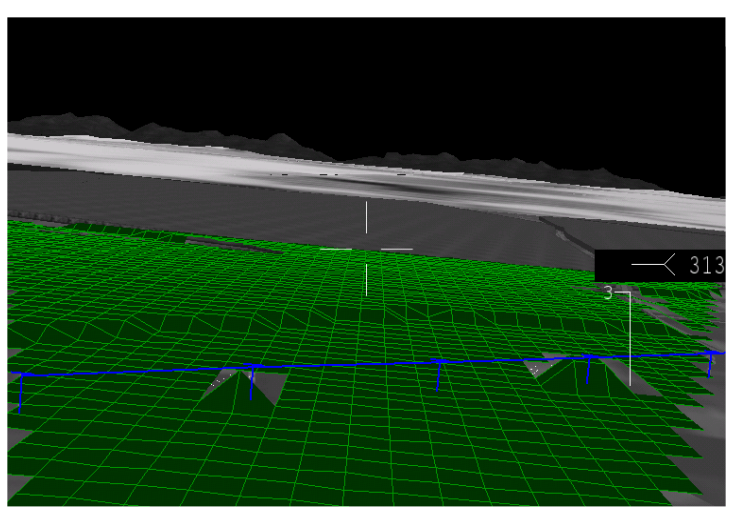

(b)

Fig. 10. Synthetic display formats developed for the Remote Piloting Station (RPS) of UA.

\section{GROUND TESTING}

Ground trials of the LOAM were performed to estimate the system detection performance in various weather and obstacle conditions to test the validity of the mathematical models used for performance calculations [4, 16, 17]. The tests were performed in various weather conditions (i.e., clear weather with $10 \leq \mathrm{V} \leq 15 \mathrm{~km}$, and light/medium/heavy rain), using a wire of known section and reflectivity (DW = $2.5 \mathrm{~cm}$ and $\rho=40 \%$ ). The sets of data collected in clear and rainy weather conditions are shown in Fig. 11. A comparison between the SNR predicted (SNRP) with $\gamma$ calculated using analytical model $(0.19 \mathrm{~km}-1 \leq \gamma \leq 0.22 \mathrm{~km}-$ 1 for clear weather and $1.23 \mathrm{~km}-1 \leq \gamma \leq 2.94 \mathrm{~km}-1$ for rainy conditions), assuming a background power of 10 $\mathrm{Watt} / \mathrm{m} 2 / \mathrm{sr} / \mu \mathrm{m}$ and $\rho=0.5$, and estimated from experimental data (SNRE), is shown in Table 1.

\section{LOAM Detection in Dry Weather}

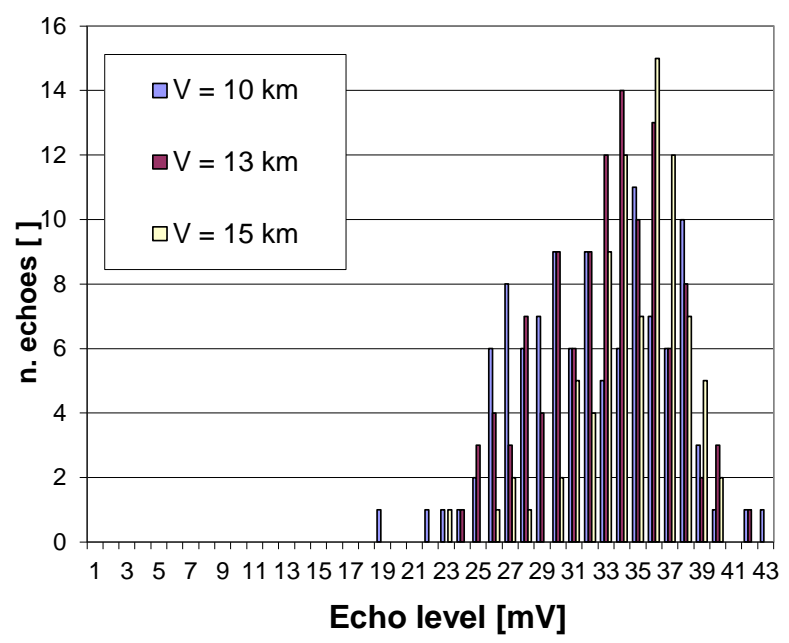

(a)

\section{LOAM Detection with Rain}

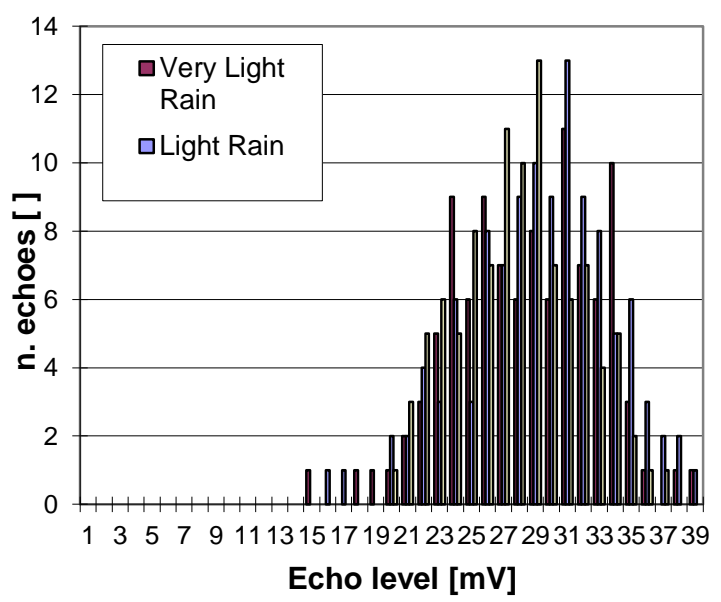

(b)

Fig. 11. LOAM detection performance in dry weather (a) and with rain (b). 
Table. 1. LOAM predicted and measured SNR's.

\begin{tabular}{|c|c|c|c|c|c|c|}
\hline & \multicolumn{3}{|c|}{ Clear Weather } & \multicolumn{3}{c|}{ Rain } \\
\hline & $\begin{array}{c}\mathrm{V}=10 \\
\mathrm{~km}\end{array}$ & $\begin{array}{c}\mathrm{V}=12.5 \\
\mathrm{~km}\end{array}$ & $\begin{array}{c}\mathrm{V}=15 \\
\mathrm{~km}\end{array}$ & Light & Medium & Heavy \\
\hline $\mathrm{SNR}_{\mathrm{P}} \times 10^{4}$ & 4.90 & 4.95 & 5.02 & 3.14 & 1.83 & 1.45 \\
\hline $\mathrm{SNR}_{\mathrm{E}} \times 10^{4}$ & 3.35 & 3.80 & 4.27 & 2.87 & 2.47 & 2.13 \\
\hline
\end{tabular}

\section{PRototype Flight Test Activities}

Table 2 shows the detection range results from the flight testing campaign, for wire obstacles of $5 \mathrm{~mm}$ in diameter, in dry weather (visibilities of $800 \mathrm{~m}, 1500 \mathrm{~m}$ and $2000 \mathrm{~m}$ ), and incidence angles of $90^{\circ}$ and $45^{\circ}$.

Table. 2. Detection range of $5 \mathrm{~mm}$ diameter cable.

\begin{tabular}{|c|c|c|c|c|}
\hline Visibility & $\begin{array}{c}\text { Incidence } \\
\text { Angle }\end{array}$ & $\begin{array}{c}\text { Analytical } \\
\text { Detection } \\
\text { Distance }\end{array}$ & $\begin{array}{c}\text { Actual } \\
\text { Detection } \\
\text { Distance }\end{array}$ & $\begin{array}{c}\text { Minimum } \\
\text { Specified } \\
\text { Detection } \\
\text { Distance }\end{array}$ \\
\hline $800 \mathrm{~m}$ & $90^{\circ}$ & $662 \mathrm{~m}$ & $727 \mathrm{~m}$ & $500 \mathrm{~m}$ \\
\hline $1500 \mathrm{~m}$ & $90^{\circ}$ & $783 \mathrm{~m}$ & $832 \mathrm{~m}$ & $560 \mathrm{~m}$ \\
\hline $2000 \mathrm{~m}$ & $90^{\circ}$ & $921 \mathrm{~m}$ & $980 \mathrm{~m}$ & $600 \mathrm{~m}$ \\
\hline $800 \mathrm{~m}$ & $45^{\circ}$ & $495 \mathrm{~m}$ & $529 \mathrm{~m}$ & $400 \mathrm{~m}$ \\
\hline $1500 \mathrm{~m}$ & $45^{\circ}$ & $553 \mathrm{~m}$ & $623 \mathrm{~m}$ & $440 \mathrm{~m}$ \\
\hline $2000 \mathrm{~m}$ & $45^{\circ}$ & $629 \mathrm{~m}$ & $657 \mathrm{~m}$ & $520 \mathrm{~m}$ \\
\hline
\end{tabular}

The flight test activities have addressed, in particular, the $\mathrm{HMI}^{2}$ and avoidance trajectory generation algorithms for rotorcraft. Two different test-bed platforms were used for these tests: NH-300 and AB-212 helicopters. For the AB212 test campaign, the LOAM CP and DU were installed in the centre of the pedestal console, in a position accessible to both pilot and co-pilot. During the test flights, a Flight Test Engineer (FTE) operated a computer, linked to the LOAM system and displaying in real-time a 3D image reconstructed using the LOAM data. All images were recorded for the successive data analysis. The LOAM range performances exceeded both the requirements and the analytical predictions $[19,20]$. Furthermore, it was verified that the LOAM history function was adequate to cover the flight envelope of relatively slow motion platforms such as helicopters and small size UAs.

\section{CONCLUSION}

This paper briefly described the hardware and software design features of the Laser Obstacle Avoidance and Monitoring (LOAM) system, which was originally designed for rotorcraft and is currently being redeveloped for smallsize Unmanned Aircraft (UA) applications. The LOAM system can be employed as one of the non-cooperative forward looking sensors (FLS) in an integrated Sense-andAvoid (SAA) architecture. The algorithms for avoidance trajectory optimisation, and determination of the overall avoidance volume (uncertainty analysis) have been presented along with a relevant simulation case study. Current research activities are addressing the integration of LOAM with other FLS and Night Vision Imaging Systems (NVIS) [21]. Display formats currently being developed for the UA remote pilot station include a Safety Line (SL) format, a Wires \& Poles (WP) format, an All Obstacles (AO) format and an Integrated LOAM/FLS (ILF) format. Additional mathematical descriptors including covariant and contravariant tensors are being adopted for avoidance volume determination, towards a unified analytical approach covering both cooperative and non-cooperative Detect-and-Avoid (DAA) applications. The possible integration of LIDAR with other UA avionic sensors is being studied and future research will address the DAA functionalities required for 4-Dimensional Trajectory Based Operations (4D-TBO) $[9,10]$.

\section{REFERENCES}

[1] R. Sabatini, "Tactical Laser Systems Performance Analysis in Various Weather Conditions", in RTO-MP-001 - E-O Propagation, Signature and System Performance under Adverse Meteorological Conditions Considering Out of Area Operations, pp. 29-1 to 29-13, NATO Research and Technology Organization (RTO), Naples, Italy, 1998.

[2] R. Sabatini, F. Guercio, and S. Vignola, "Airborne laser systems performance analysis and mission planning", in RTO-MP-046 Advanced Mission Management and Systems Integration Technologies for Improved Tactical Operations, NATO Research and Technology Organization (RTO), Florence, Italy, 1999.

[3] R. Sabatini, E. Roviaro, and M. Cottalasso, "Development of a Laser Collision Avoidance System for Helicopters: Obstacle Detection/Classification and Calculation of Alternative Flight Paths", in RTO-MP-092 - Complementarity of Ladar and Radar, NATO Research and Technology Organization (RTO), 2002.

[4] R. Sabatini and M.A. Richardson, Airborne Laser Systems Testing and Analysis: NATO Science and Technology Organization, 2010.

[5] R. Sabatini, A. Gardi, and S. Ramasamy, "A Laser Obstacle Warning and Avoidance System for Unmanned Aircraft Sense-and-Avoid", Applied Mechanics and Materials, vol. 629, pp. 355-360, 2014. DOI: 10.4028/www.scientific.net/AMM.629.355

[6] R. Sabatini, A. Gardi, S. Ramasamy, and M.A. Richardson, "A Laser Obstacle Warning and Avoidance System for Manned and Unmanned Aircraft:, in proceedings of IEEE Metrology for Aerospace (MetroAeroSpace 2014), Benevento, Italy, 2014, pp. 616-621. DOI: 10.1109/MetroAeroSpace.2014.6865998

[7] R. Sabatini, A. Gardi, and M.A. Richardson, "LIDAR Obstacle Warning and Avoidance System for Unmanned Aircraft", International Journal of Mechanical, Aerospace, Industrial and Mechatronics Engineering, vol. 8, pp. 62-73, 2014.

[8] S. Ramasamy, R. Sabatini, and A. Gardi, "Unmanned Aircraft Mission Management System for Trajectory Based Operations", in proceedings of $4^{\text {th }}$ Australasian Conference on Unmanned Systems (ACUS 2014), Melbourne, Australia, 2014. DOI: $10.13140 / 2.1 .1695 .6488$

[9] S. Ramasamy, R. Sabatini, A. Gardi, and T. Kistan, "Next Generation Flight Management System for Real-Time Trajectory Based Operations", Applied Mechanics and Materials, vol. 629, pp. 344349, 2014. DOI: 10.4028/www.scientific.net/AMM.629.344

[10] S. Ramasamy, R. Sabatini, A. Gardi, and Y. Liu, "Novel Flight Management System for Real-time 4-dimensional Trajectory Based Operations", in proceedings of AIAA Guidance, Navigation, and Control Conference 2013 (GNC 2013), Boston, MA, USA, 2013. DOI: $10.2514 / 6.2013-4763$

[11] R. Sabatini, C. Bartel, A. Kaharkar, T. Shaid, and S. Ramasamy, "Navigation and Guidance System Architectures for Small Unmanned Aircraft Applications", International Journal of 
Mechanical, Aerospace, Industrial and Mechatronics Engineering, vol. 8, pp. 733-752, 2014

[12] R. Sabatini, S. Ramasamy, A. Gardi, and L.R. Salazar, "Low-cost Sensors Data Fusion for Small Size Unmanned Aerial Vehicles Navigation and Guidance", International Journal of Unmanned Systems Engineering, vol. 1, pp. 16-47, 2013. DOI: 10.14323/ijuseng.2013.11

[13] S. Ramasamy, R. Sabatini, and A. Gardi, "Towards a Unified Approach to Cooperative and Non-Cooperative RPAS Detect-andAvoid", in proceedings of $4^{\text {th }}$ Australasian Conference on Unmanned Systems (ACUS 2014), Melbourne, Australia, 2014. DOI: 10.13140/2.1.4841.3764

[14] R.A. Hess, "Analyzing Manipulator and Feel System Effects in Aircraft Flight Control', Systems, Man and Cybernetics, IEEE Transactions on, vol. 20, no. 4, pp. 923-31, 1990.

[15] I.S. MacKenzie, "Fitts' law as a research and design tool in humancomputer interaction", Human-Computer Interaction, vol. 7, no. 1, pp. 91-139, 1992.

[16] R.E. Magdaleno and D.T. McRuer, "Experimental Validation and Analytical Elaboration Models of the Pilot's Neuromuscular Subsystem in Tracking Tasks', NASA Contractor Reports, 1971.
[17] K. Sadeghzadeh and R. Sipahi, "A Predictor-Compensator Design to Assist Human Decision-Making Process in an Air-Traffic-Control Simulator", in proceedings of ASME 2013 Dynamic Systems and Control Conference, 2013.

[18] Y. Zeyada, and R. Hess, "Modeling Human Pilot Cue Utilization with Applications to Simulator Fidelity Assessment", Journal of aircraft, vol. 37, no. 4, pp. 588-97, 2000.

[19] R. Sabatini and M.A. Richardson, "Novel Atmospheric Extinction Measurement Techniques for Aerospace Laser System Applications", Infrared Physics and Technology, vol. 56, pp. 30-50, 2013. DOI: 10.1016/j.infrared.2012.10.002

[20] R. Sabatini, M.A. Richardson, H. Jia, and D. Zammit-Mangion, "Airborne Laser Systems for Atmospheric Sounding in the Near Infrared", in proceedings of SPIE 8433, Laser Sources and Applications, Photonics Europe 2012, Brussels, Belgium, 2012. DOI: 10.1117/12.915718

[21] R. Sabatini, M.A. Richardson, M. Cantiello, M. Toscano, and P. Fiorini, "A Novel Approach to Night Vision Imaging Systems Development, Integration and Verification in Military Aircraft", Aerospace Science and Technology, 2013. DOI: 10.1016/j.ast.2013.08.021 